the transcendentals corresponding to $\pi$ and $e$ in the $n$th process are algebraic numbers borrowed from the $(n-1)$ th and $(n+1)$ th processes respectively. The totality of numbers may thus be conceived as arranged in an infinite number of strata, the ordinary algebraic numbers forming a single stratum.

With the extension of the differentiation process, and the stratification of the number body, all the results of the ordinary calculus, for example Taylor's theorem, can be expressed in terms of any two consecutive processes.

Evanston, ILL.

Thomas F. Holgate, Secretary of the Section.

\title{
THE VALUE OF $\int_{0}^{\pi / 2}(\log 2 \cos \varphi)^{m} \varphi^{n} d \varphi$
}

BY PROFESSOR F. MORLEY.

(Read before the American Mathematical Society, April 29, 1899.)

The integral in question, in which $m$ is any positive integer, and $n$ is any even positive integer or zero, is given in effect, for the cases $m=1, n=0$, in Williamson's Integral Calculus, $\$ 118$ of the second edition, being taken from a paper by H. G. (presumably Harvey Goodwin, Bishop of Carlisle) in volume 3 of the Quarterly Journal of Mathematics. Further it is given in effect, for the case $m=2, n=0$, in Wolstenholme's Problems, p. 332 of the second edition. It seems worth while to show how it can be expressed in general, in terms of the constants $s_{r}=\sum_{1}^{\infty} 1 / n^{r}$, which may be regarded as known.

We know that when $p$ and $q$ are real

$$
1+\frac{p \cdot q}{1 \cdot 1}+\frac{p(p-1) q(q-1)}{1 \cdot 2 \cdot 1 \cdot 2}+\cdots=\frac{\Gamma(1+p+q)}{\Gamma(1+p) \Gamma(1+q)}
$$

when $p+q>-1$ (Forsyth, Differential equations, p. 197). But the left member is the constant term in the product of the series

and

$$
(1+x)^{p}=1+p x+\cdots
$$

$$
(1+1 / x)^{q}=1+q / x+\cdots .
$$


We suppose $p$ and $q$ positive, so that both series are absolutely convergent when $|x|=1$. We may then multiply the series and get a Laurent series for

Hence

$$
(1+x)^{p}(1+1 / x)^{q} \text {. }
$$

$$
\frac{1}{2 i \pi} \int_{|x|=1}(1+x)^{p}(1+1 / x)^{q} \frac{d x}{x}=\frac{\Gamma(1+p+q)}{\Gamma(1+p) \Gamma(1+q)} .
$$

The path of integration shall be from -1 positively round the circle; the point -1 being a branch point of the integral, but not an infinity.

Writing $x=\exp 2 i \varphi$, the integral becomes

Let

$$
\frac{1}{\pi} \int_{-\pi / 2}^{\pi / 2}(2 \cos \varphi)^{p+q} \exp i(p-q) \varphi d \varphi .
$$

then

$$
p+q=2 \alpha, \quad p-q=2 \beta
$$

$$
\begin{gathered}
\frac{1}{\pi} \int_{-\pi / 2}^{\pi / 2} \exp \{2 \alpha \log (2 \cos \varphi)+2 i \beta \varphi\} d \varphi \\
=\frac{\Gamma(1+2 \alpha)}{I^{\prime}(1+\alpha+\beta) \Gamma(1+\alpha-\beta)} .
\end{gathered}
$$

Both sides can be developed in the form

$$
\sum \Sigma C_{m, n} a^{m} \beta^{n}
$$

the left directly by the exponential theorem, giving

$$
C_{m, n}=\frac{2^{m+n}}{\pi m ! n !} \int_{-\pi / 2}^{\pi / 2}(\log 2 \cos \varphi)^{m}(i \varphi)^{n} d \varphi,
$$

while the right, obtained from the formula

is

$$
\log \Gamma(1+n)=-r n+\sum_{2}^{\infty}(-)^{r} \frac{s_{r}}{r} x^{r}
$$

$$
\exp \left[\sum_{2}^{\infty}(-)_{r}^{r} \frac{{ }^{\varepsilon}}{r}\left\{(2 \alpha)^{r}-(\alpha+\beta)^{r}-(\alpha-\beta)^{r}\right\}\right] .
$$

Hence the result: The integral

$$
\frac{2}{\pi} \int_{0}^{\pi / 2}(\log 2 \cos \varphi)^{m} \varphi^{n} d \varphi
$$

where $m$ is any positive integer, $n$ any positive even integer or 0 , is 


$$
\begin{gathered}
(-)^{n / 2} \frac{m ! n !}{2^{m+n}} \times \text { the coefficient of } \alpha^{m} \beta^{n} \text { in } \\
\exp \left[\sum_{2}^{\infty}(-)^{r} \frac{s_{r}}{r}\left\{(2 \alpha)^{r}-(\alpha+\beta)^{r}-(\alpha-\beta)^{r}\right\}\right] .
\end{gathered}
$$

This generating function is, to a few terms,

$$
\begin{gathered}
\exp \left[s_{2}\left(\alpha^{2}-\beta^{2}\right)-2 \alpha s_{3}\left(\alpha^{2}-\beta^{2}\right)\right. \\
\left.+\frac{1}{2} s_{4}\left(7 \alpha^{4}-6 \alpha^{2} \beta^{2}-\beta^{4}\right)-2 \alpha s_{5}\left(3 \alpha^{4}-2 \alpha^{2} \beta^{2}-\beta^{4}\right)+\right]
\end{gathered}
$$

or

$$
\begin{aligned}
& 1+s_{2}\left(\alpha^{2}-\beta^{2}\right)-2 s_{3}\left(\alpha^{3}-\alpha \beta^{2}\right) \\
& \quad+\frac{s_{2}{ }^{2}+7 s_{4}}{2} \alpha^{4}-\left(s_{2}{ }^{2}+3 s_{4}\right) \alpha^{2} \beta^{2}+\frac{s_{2}{ }^{2}-s_{4}}{2} \beta^{4} \\
& \quad-2\left(s_{2} s_{3}+3 s_{5}\right) \alpha^{5}+4\left(s_{2} s_{3}+s_{5}\right) \alpha^{3} \beta^{2}-2\left(s_{2} s_{3}-s_{5}\right) \alpha \beta^{4}+\cdots
\end{aligned}
$$

Thus, in tabular form, a few values for

$$
\frac{2}{\pi} \int_{0}^{\pi / 2}(\log 2 \cos \varphi)^{m} \varphi^{n} d \varphi
$$

are :

\begin{tabular}{c|c|c|c}
\hline \hline$n=$ & 0 & 2 & 4 \\
$m=0 *$ & 1 & $\frac{1}{2} s_{2}$ & $\frac{3}{4}\left(s_{2}^{2}-s_{4}\right)$ \\
1 & 0 & $-\frac{1}{2} s_{3}$ & $-\frac{3}{2}\left(s_{2} s_{3}-s_{5}\right)$ \\
2 & $\frac{1}{2} s_{2}$ & $\frac{1}{4}\left(s_{2}^{2}+3 s_{4}\right)$ & \\
3 & $-\frac{3}{2} s_{3}$ & $-\frac{3}{2}\left(s_{2} s_{3}+s_{5}\right)$ & \\
4 & $\frac{3}{4}\left(s_{2}^{2}+7 s_{4}\right)$ & & \\
5 & $-\frac{15}{2}\left(s_{2} s_{3}+3 s_{5}\right)$ & & \\
\hline
\end{tabular}

\section{ON THE ALGEBRAIC POTENTIAL CURVES.}

BY DR. EDWARD KASNER.

(Read before the American Mathematical Society, February 23, 1901.)

THE object of this paper is to derive the characteristic geometric properties of a class of curves which are of in-

* The row for which $m=0$ is of course merely a verification, leading to the known values

$$
s_{2}=\pi^{2} / 6, \quad s_{4}=\pi^{4} / 90 \text {. }
$$

